\title{
From the Hubbard to the PPP Model $^{\dagger}$
}

\author{
Thomas G. Schmalz \\ Texas A\&M University at Galveston, P. O. Box 1675, Galveston, Texas 77553 USA \\ (E-mail:schmalzt@tamug.edu)
}

RECEIVED JUNE 10, 2013; REVISED SEPTEMBER 5, 2013; ACCEPTED SEPTEMBER 6, 2013

\begin{abstract}
This paper presents an extension of the Hubbard Model to Pariser-Parr-Pople form. Although the Hubbard model contains most of the essentials of chemical bonding, it is unable to describe excited states with separated charges, such as the lowest ${ }^{1} \mathrm{~B}_{\mathrm{u}}$ states of linear polyenes. The PPP model adds longrange electron-electron repulsions to the Hubbard model to remedy this defect. If the long range repulsion integrals are assumed to follow a standard form, all parameters in the model can be evaluated exactly from high accuracy $a b$ initio computations on stretched ethlyene. This yields a model based on the MatagaNishimoto form for the long-range integrals which gives excellent agreement with both excitation energies and ground-state bond lengths, but with a significantly smaller value of the one center electron repulsion $U$ than is usually assumed. A major conclusion of this work is that the exact form of the long-range integrals is not so important, but that the value of the one center integral $U$ must be chosen smaller than traditional values. The PPP-MN model is recommended for applications because it contains no adjustable parameters, with all parameter values determined directly from ab initio results. (doi: 10.5562/cca2297)
\end{abstract}

Keywords: pi-electron systems, Pariser-Parr-Pople model, Hubbard model, excitation energies, equilibrium bond lengths

\section{INTRODUCTION}

In a recent paper $^{1}$ we argued that the Hubbard Model ${ }^{2,3}$ provides the simplest and easiest to understand quantitative model of basic chemical bonding effects. The model includes on-site and nearest-neighbor terms of the Hückel model to account for the formation of chemical bonds and on-site and nearest-neighbor electron repulsion terms to account for electron correlation. The Hubbard model is widely used in the physics literature to treat phenomena as diverse as high-temperature superconductivity, ${ }^{4}$ conducting polymers, ${ }^{5}$ and molecular magnetism. ${ }^{6}$ All parameters in the model may be evaluated from high-quality $a b$ initio calculations on two-site fragments of whatever type of system is being considered. We illustrated ${ }^{1}$ the parameterization and use of the model for the pi electrons in conjugated hydrocarbons, and showed that both the ground state geometry and the lowlying electronic excitation spectrum of a few small test molecules may generally be predicted quite accurately.

However, we also noted one situation where the model fails. ${ }^{1}$ The Hubbard model correctly positions ionic excited states (those composed dominantly of determinants in the atomic orbital basis having separated charges) with respect to covalent states (those, such as the ground state, composed dominantly of determinants in the atomic orbital basis with one electron per site). But because it lacks long-range electron-electron repulsion terms, it is incapable of properly positioning states with large distances between the positively and negatively charged sites with respect to those in which the dominant determinants have the charges on adjacent sites. A particularly obvious example of this deficiency occurs with the ${ }^{1} B_{u}$ excited state in polyenes such as butadiene and hexatriene. Although in long polyenes the lowest singlet excited state is the optically forbidden socalled homopolar ${ }^{1} \mathrm{~A}_{\mathrm{g}}$ state, in butadiene and likely also hexatriene the strongly optically allowed ${ }^{1} \mathrm{~B}_{\mathrm{u}}$ state lies lower, with the cross-over probably occurring near octatetraene. ${ }^{7}$ The ${ }^{1} \mathrm{~B}_{\mathrm{u}}$ state is known as the charge transfer state, and it has important contributions from determinants with the positive charge on one end of the molecule and the negative charge on the other. Accordingly, as noted in our previous paper, ${ }^{1}$ these states are predicted very poorly by the Hubbard model.

The solution to this deficiency is to extend the model Hamiltonian from the Hubbard to the PariserParr-Pople (PPP) form. ${ }^{8}$ The PPP Hamiltonian is of exactly the same form as the Hubbard Hamiltonian, except that the two-center electron repulsion terms are

\footnotetext{
$\dagger$ Dedicated to Professor Douglas Jay Klein on the occasion of his $70^{\text {th }}$ birthday.
} 
allowed to act between any two sites, not just nearest neighbors. Because there may be many such long-range electron repulsions, these are usually found from an interpolation formula rather than from direct calculation. The PPP model was developed in the 1950's to describe excited states of conjugated hydrocarbons, and its use for this purpose continues unabated ${ }^{9-11}$ since it can be applied to larger molecules beyond the reach of modern $a b$ initio methods. More recently it has played a critical role in understanding dimerization in onedimensional conjugated polymers, ${ }^{12-15}$ and has been applied to the electronic structure of fullerenes, ${ }^{16}$ and to a variety of other problems including organic chargetransfer salts. ${ }^{17}$ In the next section the model Hamiltonians will be presented in detail and the evaluation of the long-range electron repulsion terms from standard interpolation formulas discussed. Applications of the model to electronic excitations and to ground state geometries will be presented in the following sections.

\section{MODEL HAMILTONIANS}

In second quantized form the Hubbard model Hamiltonian can be written as

$$
\begin{aligned}
& H^{\mathrm{Hub}}=\sum_{i} t_{i i} E_{i i}+\sum_{i j} t_{i j} E_{i j}+\sum_{i} U_{i} n_{i \alpha} n_{i \beta}+ \\
& \frac{1}{2} \sum_{i j} V_{i j}\left(Z_{i}-n_{i}\right)\left(Z_{j}-n_{j}\right)+V_{c}
\end{aligned}
$$

where $E_{i j}=\sum_{\sigma=\alpha, \beta}\left(a_{i \sigma}^{+} a_{j \sigma}+a_{j \sigma}^{+} a_{i \sigma}\right), n_{i \sigma}=a_{i \sigma}^{+} a_{i \sigma}$ is the number operator for electrons of spin $\sigma$ on site $i$, and $a_{i \sigma}^{+}\left(a_{i \sigma}\right)$ are creation (annihilation) operators for an electron of spin $\sigma$ in orbital $i$. With the double sum limited to nearest-neighbor sites and the identification $t_{i i}=$ $\alpha_{i}, t_{i j}=\beta_{i j}$ the first two terms of Equation (1) are identical to the Hückel model Hamiltonian. The third term of Equation (1) adds an effective repulsion $U_{i}$ for two electrons which occupy the same atomic orbital, while the fourth term introduces an electron repulsion $V_{i j}$ for two electrons on nearest-neighbor sites. Finally, the last term adds a potential $V_{c}$ to account for the effects of the nuclei and electrons not considered explicitly in the model. $V_{c}$ may depend on the positions of the nuclei but is assumed to be independent of the distribution of electrons over the active orbitals of the model. For systems in which all of the atoms are chemically identical, the first term of Equation (1) simply adds a constant to the energy of all states at all geometries. Formally it may be set to zero and incorporated into $V_{c}$, and we assume that this has been done in the following discussion.

The PPP model differs from Equation (1) only in the fourth term, where $V_{i j}$ is replaced with $\gamma_{i j}$ and the sum is allowed to run over all pairs of sites, not just nearest neighbors. This yields, with the first term dropped,

$$
\begin{aligned}
& H^{\mathrm{PPP}}=\sum_{i j} t_{i j} E_{i j}+\sum_{i} U_{i} n_{i \alpha} n_{i \beta}+ \\
& \frac{1}{2} \sum_{i j} \gamma_{i j}\left(Z_{i}-n_{i}\right)\left(Z_{j}-n_{j}\right)+V_{c}
\end{aligned}
$$

Two interpolation formulas for the $\gamma_{i j}$ 's have frequently been used. In semiempirical applications of the PPP model $\gamma_{i j}$ is often assumed to be related to the value of $U$ (assumed here to be the same for all sites) by either the Mataga-Nishimoto formula, ${ }^{18}$

$$
\gamma_{i j}^{\mathrm{MN}}\left(R_{i j}\right)=\frac{1}{1 / U+R_{i j}}
$$

or the Ohno formula, ${ }^{19}$

$$
\gamma_{i j}^{\mathrm{OH}}\left(R_{i j}\right)=\frac{1}{\sqrt{1 / U^{2}+R_{i j}^{2}}}
$$

In Equations (3) and (4) $\mathrm{R}_{\mathrm{ij}}$ is the distance between sites $i$ and $j$ and all quantities are in atomic units. These formulas are simply interpolation formulas between a one-center electron-electron repulsion integral and the asymptotic Coulombic form of a two-center electronelectron repulsion integral.

Once the form of $\gamma_{i j}$ is chosen, the $\gamma_{i j}$ 's can be evaluated by requiring Equation (3) or (4) to give the value of $U-V=U-\gamma_{12}$ from the $a b$ initio calculations on stretched ethylene presented in Ref. (1) at some convenient reference point. The choice made here is $R_{12}=$ $1.40 \AA$, which is typical of the $\mathrm{C}-\mathrm{C}$ bond length in aromatic rings, and is midway between the extreme $\mathrm{C}-\mathrm{C}$ bond lengths $(1.33 \AA-1.47 \AA)$ in linear conjugated polyenes. The results are $U^{\mathrm{MN}}=8.325 \mathrm{eV}$ and $U^{\mathrm{OH}}=$ $11.344 \mathrm{eV}$. It should be emphasized that, once a functional form for the $\gamma$ 's is chosen, this procedure involves no adjustable parameters. All parameters of the model $t_{i j}, U$, and $\gamma_{i j}$ - are completely determined from the accurate $a b$ initio results for ethylene.

\section{EXCITATION ENERGIES}

Transition energies to the low-lying excited states of three test molecules, trans butadiene, all trans hexatriene, and benzene, computed from the Hubbard model and the PPP model with the Mataga-Nishimoto (MN) and $\mathrm{Ohno}(\mathrm{OH})$ forms for the long-range electron repulsions, are compared in Table 1. The PPP calculations were performed with a modified version of the Hubbard program used in Ref. (1) written by the author. All calculations are at the experimental geometry, ${ }^{20}$ and except for the $2^{1} \mathrm{~A}_{\mathrm{g}}$ states of the polyenes which are unknown, compared to the experimental excitation energies. ${ }^{21}$ As can be seen, excitation energies computed from the PPP model using the Mataga-Nishimoto formula are in 
Table 1. Low energy excitation energies ${ }^{(a)}$ for trans butadiene, all trans hexatriene, and benzene computed from the Hubbard model and the PPP model with the Mataga-Nishimoto (MN) and $\mathrm{Ohno}(\mathrm{OH})$ forms for long range electron repulsions

\begin{tabular}{|c|c|c|c|c|}
\hline$U$ & Expt. $^{(b)}$ & $\begin{array}{c}\text { Hubbard } \\
5.0 \mathrm{eV}\end{array}$ & $\begin{array}{l}\text { PPP-MN } \\
8.325 \mathrm{eV}\end{array}$ & $\begin{array}{c}\text { PPP-OH } \\
11.344 \mathrm{eV}\end{array}$ \\
\hline \multicolumn{5}{|c|}{ Butadiene } \\
\hline $1^{1} \mathrm{~B}_{\mathrm{u}}$ & 5.92 & 5.67 & 5.94 & 6.37 \\
\hline $2^{1} \mathrm{~A}_{\mathrm{g}}$ & - & 6.27 & 6.25 & 6.28 \\
\hline $1^{3} \mathrm{~B}_{\mathrm{u}}$ & 3.20 & 3.18 & 3.15 & 3.21 \\
\hline $1^{3} \mathrm{~A}_{\mathrm{g}}$ & 5.08 & 4.95 & 4.92 & 4.89 \\
\hline \multicolumn{5}{|c|}{ Hexatriene } \\
\hline $1^{1} B_{u}$ & $5.13,4.93^{(\mathrm{c})}$ & $4.47 *$ & 4.91 & 5.45 \\
\hline $2^{1} \mathrm{~A}_{\mathrm{g}}$ & - & 5.09 & 5.11 & 5.16 \\
\hline $1^{3} \mathrm{~B}_{\mathrm{u}}$ & 2.61 & 2.55 & 2.52 & 2.58 \\
\hline $1^{3} \mathrm{~A}_{\mathrm{g}}$ & 4.11 & 4.15 & 4.13 & 4.14 \\
\hline \multicolumn{5}{|l|}{ Benzene } \\
\hline $1{ }^{1} \mathrm{~B}_{2 \mathrm{u}}$ & 4.90 & 4.97 & 4.93 & 4.93 \\
\hline $1^{1} \mathrm{~B}_{1 \mathrm{u}}$ & 6.20 & 6.31 & 6.54 & 6.14 \\
\hline $1{ }^{1} \mathrm{E}_{1 \mathrm{u}}$ & 6.94 & 6.98 & 6.94 & $7.68^{*}$ \\
\hline $1^{3} \mathrm{~B}_{1 \mathrm{u}}$ & 3.94 & 4.16 & 4.14 & 4.17 \\
\hline $1^{3} \mathrm{E}_{1 \mathrm{u}}$ & 4.76 & 4.93 & 4.89 & 4.98 \\
\hline $1^{3} \mathrm{~B}_{2 \mathrm{u}}$ & 5.60 & 6.06 & $5.96^{*}$ & 6.18 \\
\hline $\mathrm{RMS}^{(\mathrm{d})}$ & & 0.26 & 0.18 & 0.34 \\
\hline $\mathrm{MAD}^{(\mathrm{e})}$ & & 0.63 & 0.36 & 0.74 \\
\hline
\end{tabular}

(a) All excitation energies are expressed in electron volts, $\mathrm{eV}$.

(b) Experimental results taken from Ref. (21).

(c) $5.03 \mathrm{eV}$ used to compute deviations.

(d) Root Mean Square deviation with respect to experiment. ${ }^{1} \mathrm{~A}_{\mathrm{g}}$ states of butadiene and hexatriene omitted.

${ }^{(e)}$ Maximum Absolute Deviation with respect to experiment. Maximum marked with *.

excellent agreement with experiment. The large errors in the ${ }^{1} B_{u}$ states of butadiene and hexatriene are corrected, and the root mean square error is only $0.18 \mathrm{eV}$. In fact the only apparent "problem" with these results lies in the value of the one center electron repulsion integral $U=8.32 \mathrm{eV}$. This is much smaller than the commonly accepted semiempirical value which is usually cho$\operatorname{sen}^{9,15,22,23}$ in the range $10.84-11.16 \mathrm{eV}$.

By contrast the PPP-Ohno formula yields a value of $U=11.34 \mathrm{eV}$, in much better agreement with the semiempirical values, but gives an unsatisfactory prediction of the spectra. The ${ }^{1} \mathrm{~B}_{\mathrm{u}}$ states of butadiene and hexatriene are predicted to lie above the ${ }^{1} \mathrm{~A}_{\mathrm{g}}$ states, and several other ionic states show large errors. The rms error of $0.34 \mathrm{eV}$ is almost twice that of the PPP-MN calculation, and is larger even than the rms error of 0.26 $\mathrm{eV}$ from the original Hubbard model. However, if the long range coulomb integrals are viewed as semiempirical parameters it is not really necessary for the extrapo-
Table 2. Low energy excitation energies ${ }^{(a)}$ for trans butadiene, all trans hexatriene, and benzene computed from the PPP model with the Mataga-Nishimoto (MN) and optimized Ohno (OHopt) forms for long range electron repulsions

\begin{tabular}{|c|c|c|c|c|}
\hline $\begin{array}{c}U \\
U_{\text {extrap. }}\end{array}$ & Expt. $^{(\mathrm{b})}$ & $\begin{array}{l}\text { PPP-MN } \\
\text { Expt. } \\
\text { Geom. }{ }^{\left({ }^{c}\right)} \\
8.325 \mathrm{eV}\end{array}$ & $\begin{array}{l}\text { PPP-OHopt } \\
\text { Expt. } \\
\text { Geom. }{ }^{(c)} \\
9.30 \mathrm{eV} \\
6.64 \mathrm{eV}\end{array}$ & $\begin{array}{c}\text { PPP-MN } \\
\text { Model } \\
\text { Geom. }^{(\mathrm{d})}\end{array}$ \\
\hline \multicolumn{5}{|c|}{ Butadiene } \\
\hline $1{ }^{1} \mathrm{~B}_{\mathrm{u}}$ & 5.92 & 5.94 & 6.03 & 5.92 \\
\hline $2^{1} \mathrm{~A}_{\mathrm{g}}$ & - & 6.25 & 6.24 & 6.23 \\
\hline $1^{3} \mathrm{~B}_{\mathrm{u}}$ & 3.20 & 3.15 & 3.15 & 3.13 \\
\hline $1^{3} \mathrm{~A}_{\mathrm{g}}$ & 5.08 & 4.92 & 4.91 & 4.93 \\
\hline \multicolumn{5}{|c|}{ Hexatriene } \\
\hline $1{ }^{1} \mathrm{~B}_{\mathrm{u}}$ & $5.13,4.93^{(\mathrm{e})}$ & 4.91 & 5.06 & 4.87 \\
\hline $2^{1} \mathrm{~A}_{\mathrm{g}}$ & - & 5.11 & 5.11 & 4.99 \\
\hline $1^{3} \mathrm{~B}_{\mathrm{u}}$ & 2.61 & 2.52 & 2.52 & 2.47 \\
\hline $1^{3} \mathrm{~A}_{\mathrm{g}}$ & 4.11 & 4.13 & 4.12 & 4.02 \\
\hline \multicolumn{5}{|l|}{ Benzene } \\
\hline $1{ }^{1} \mathrm{~B}_{2 \mathrm{u}}$ & 4.90 & 4.93 & 4.92 & 4.95 \\
\hline $1{ }^{1} \mathrm{~B}_{1 \mathrm{u}}$ & 6.20 & 6.54 & $6.58^{*}$ & 6.55 \\
\hline $1^{1} \mathrm{E}_{1 \mathrm{u}}$ & 6.94 & 6.94 & 6.96 & 6.96 \\
\hline $1^{3} \mathrm{~B}_{1 \mathrm{u}}$ & 3.94 & 4.14 & 4.13 & 4.15 \\
\hline $1^{3} E_{1 u}$ & 4.76 & 4.89 & 4.88 & 4.90 \\
\hline $1^{3} \mathrm{~B}_{2 \mathrm{u}}$ & 5.60 & $5.96^{*}$ & 5.95 & $5.98^{*}$ \\
\hline $\operatorname{RMS}^{(\mathrm{f})}$ & & 0.18 & 0.18 & 0.19 \\
\hline $\operatorname{MAD}^{(g)}$ & & 0.36 & 0.38 & 0.38 \\
\hline
\end{tabular}

(a) All excitation energies are expressed in electron volts, $\mathrm{eV}$.

(b) Experimental results taken from Ref. (21).

(c) At the experimental geometry of Ref. (20).

(d) At the model equilibrium geometry.

(e) $5.03 \mathrm{eV}$ used to compute deviations.

(f) Root Mean Square deviation with respect to experiment. ${ }^{1} \mathrm{~A}_{\mathrm{g}}$ states of butadiene and hexatriene omitted.

${ }^{\text {(g) }}$ Maximum Absolute Deviation with respect to experiment. Maximum marked with *.

lation formula for the $\gamma$ 's to predict the one center value of $U$. Since the difference $U-\gamma_{12}$ is known accurately from the $a b$ initio results for ethylene, it is sufficient that it predict the longer range $\gamma$ 's from $\gamma_{12}$, while $U$ can be considered an adjustable parameter. If this freedom is invoked with the Mataga-Nishimoto formula little improvement occurs, but for the Ohno formula the changes are quite dramatic. The optimum value of $U$ drops to $9.30 \mathrm{eV}$, while the effective value of the extrapolation parameter in Equation (4), which will be denoted by $U_{\text {extrap., }}$ is even smaller at $U_{\text {extrap. }}=6.64 \mathrm{eV}$.

The predictions of the PPP-MN and the PPP model with the optimized Ohno formula, denoted PPPOHopt, are compared in Table 2 in the columns marked experimental geometry. What is apparent is that not 
only is the same overall quality obtained by both models, but that the two virtually coincide transition by transition. The obvious conclusion is that the exact form of the long range $\gamma$ 's is unimportant. Any reasonable decaying function will do. But the value of the one center electron repulsion needs to be significantly smaller than the traditional semiempirical value near $11 \mathrm{eV}$.

The semiempirical values of $U$ trace back to an argument originally given by Pariser $^{24}$, who by considering the reaction

$$
\mathrm{C}+\mathrm{C} \longrightarrow \mathrm{C}^{+}+\mathrm{C}^{-}
$$

concluded that $U$ should be equal to the difference between the valence state (i.e., $\mathrm{sp}^{2}$ hybridized) ionization potential and electron affinity of carbon. The valence state ionization potential $^{25}$ is believed to be about 11.15 $\mathrm{eV}$, while the less well known electron affinity is believed to fall in the range $0-0.3 \mathrm{eV}$. However this argument neglects any screening due to the extended nature of the sigma bond framework of conjugated hydrocarbons. While I cannot provide any direct evidence for the magnitude of this effect, it seems the most reasonable explanation for the smaller values of $U$ required by the models presented here.

\section{BOND LENGTHS}

Differences in predicted ground state geometry between the PPP and Hubbard models are expected to be small since the Hubbard model already provides a good description of primarily covalent states such as the ground state. Table 3 compares bond lengths in the three test molecules considered in the previous section as computed from the Hubbard, PPP-MN, and PPP-OHopt Hamiltonians. As expected the predictions from all three model Hamiltonians are almost identical. In general they are in very good agreement with experimentally measured bond lengths, except for the end double bond in hexatriene.

All theoretical methods of which I am aware produce a monotonic lengthening of the end double bond in the series ethylene, butadiene, hexatriene, reflecting increased electron delocalization through the longer molecular chain. ${ }^{26}$ Accordingly in Ref. (1) the opinion was expressed that the experimental value of the end bond length in hexatriene, which is shorter than that of butadiene, was probably in error. This value apparently traces back to the usually reliable experimental work of Haugen and Traetteberg ${ }^{27}$ in 1966. However recent (though still preliminary) work on $\mathrm{cis}^{28}$ and trans ${ }^{29}$ hexatriene, while showing the expected sequence for the cis isomer, also finds a shortening of the end bond in the trans isomer, so this remains an open question.
Table 3. Bond lengths ${ }^{(a)}$ for trans butadiene, all trans hexatriene, and benzene computed from the Hubbard model and the PPP model with the Mataga-Nishimoto (MN) and optimized Ohno (OHopt) forms for long range electron repulsion

\begin{tabular}{|c|c|c|c|c|}
\hline $\begin{array}{c}U \\
U_{\text {extrap. }}\end{array}$ & Expt. $^{(b)}$ & $\begin{array}{c}\text { Hubbard } \\
5.0 \mathrm{eV}\end{array}$ & $\begin{array}{l}\text { PPP-MN } \\
8.325 \mathrm{eV}\end{array}$ & $\begin{array}{c}\text { PPP-OHopt } \\
9.3 \mathrm{eV} \\
6.64 \mathrm{eV} \\
\end{array}$ \\
\hline \multicolumn{5}{|l|}{ Butadiene } \\
\hline double & 1.349 & 1.350 & 1.349 & 1.349 \\
\hline single & 1.467 & 1.461 & 1.461 & 1.462 \\
\hline \multicolumn{5}{|l|}{ Hexatriene } \\
\hline end & 1.337 & 1.352 & 1.351 & 1.351 \\
\hline single & 1.458 & 1.454 & 1.456 & 1.457 \\
\hline middle & 1.368 & 1.362 & 1.361 & 1.361 \\
\hline \multicolumn{5}{|l|}{ Benzene } \\
\hline aromatic & 1.399 & 1.397 & 1.397 & 1.397 \\
\hline
\end{tabular}

(a) Bond lengths are expressed in $\AA$.

(b) Experimental results taken from Ref. (20).

Transition energies recomputed at the model geometry for the PPP-MN model are shown in the last column of Table 2. For butadiene and benzene, where the model geometry is very close to experiment, shifts of only $0.01-0.02 \mathrm{eV}$ are observed. For hexatriene, where the bond length of the end bond is significantly different, shifts of up to $0.11 \mathrm{eV}$ are observed for the $\mathrm{A}_{\mathrm{g}}$ states. The ${ }^{1} \mathrm{~A}_{\mathrm{g}}$ state is not known experimentally, but the agreement for the ${ }^{3} \mathrm{~A}_{\mathrm{g}}$ state becomes somewhat poorer. The PPP-ONopt model (not shown) tracks the PPP-MN model exactly. That is, the shifts of each transition energy from the experimental to the model geometry are identical.

\section{CONCLUSION}

This paper has presented an extension of the $a b$ initioderived Hubbard Model of Ref. (1) to Pariser-ParrPople form. Although the Hubbard model contains most of the essentials of chemical bonding, it is unable to describe excited states with separated charges, such as the lowest ${ }^{1} \mathrm{~B}_{\mathrm{u}}$ states of linear polyenes. The PPP model adds long-range electron-electron repulsions to the Hubbard model to remedy this defect. If the long range repulsion integrals are assumed to follow a standard form such as that of Mataga-Nishimoto ${ }^{18}$ or Ohno, ${ }^{19}$ all parameters in the model can be evaluated exactly from high accuracy $a b$ intio computations on stretched ethlyene. ${ }^{1}$ This yields a model based on the MN form for the long-range integrals which gives excellent agreement with both excitation energies and ground-state bond lengths, but with a significantly smaller value of the one center electron repulsion $U$ than is usually assumed. By contrast the Ohno form for the long-range 
integrals agrees poorly with several experimental excitation energies. This disagreement can be remedied by allowing the one center electron repulsion integral to be a variable parameter, giving a model which virtually duplicates the MN results, but with again a much smaller value of $U$. A major conclusion of this work is that the exact form of the long-range integrals is not so important, but that the value of the one center integral $U$ derived from accurate $a b$ initio calculations is much smaller than traditional values. The PPP-MN model is recommended for applications because it contains no adjustable parameters, with all parameter values determined directly from $a b$ initio results.

It should be mentioned that the models developed here and in Ref. (1) are similar in spirit both to the PPP model of Ref. (9) and to the earlier Hubbard model of Malrieu and co-workers. ${ }^{30}$ In Ref. (1) we attempted to extract all model parameters as accurately as is possible from state-of-the-art $a b$ initio calculations on ethylene, and the PPP-MN model developed here uses only that same input. On the other hand, we have treated the one center electron-electron repulsion $U$ as a constant, depending only on the nature of the $2 \mathrm{p} \pi$ orbital. In Ref. (9) $U$ is viewed as reflecting the influence of its nearestneighbor surroundings, introducing a possibly useful additional degree of freedom, but one whose effects cannot be determined from calculations on ethylene alone. The spectrum of ethylene is completely determined by the difference $U-V$, where $V$ is the nearestneighbor electron-electron repulsion, so it is impossible to assign variations in the difference to either $U$ or $V$ separately.

Acknowledgements. This paper is dedicated to Professor Douglas Klein on his seventieth birthday, who has been a valuable collaborator for over thirty years and from whom I have benefitted from many discussions on the nature of pielectron systems. I also wish to acknowledge the assistance of Professor Larry Griffin in checking $a b$ initio excitation energies. This work was partially supported by the Robert A. Welch Foundation through Grant No. BD-0046.

\section{REFERENCES}

1. T. G. Schmalz, L. Serrano-Andres, V. Sauri, M. Merchan, and J. M. Oliva, J. Chem. Phys. 135 (2011) 194103.

2. J. Hubbard, Proc. R. Soc. London Ser. A 276 (1963) 238; 281 (1964) 401.

3. F. A. Matsen, Acc. Chem. Res. 11 (1978) 387; M. A. Fox and F. A. Matsen, J. Chem. Educ. 62 (1985), 367; 62 (1985) 477; 62 (1985) 551.
4. J. C. Phillips, High $T_{C}$ Superconductors, Academic Press, San Diego, 1989.

5. W. P. Su, Phys. Rev. Lett. 74 (1995) 1167.

6. V. I. Anisimov, F. Aryasetiawan, and A. I. Lichtenstein, J. Phys. Condens. Matter 9 (1997) 767; C. Cao, S. Hill, and H. P. Cheng, Phys. Rev. Lett. 100 (2008) 167206.; E. Scrivan and B. J. Powell, J. Chem. Phys. 130 (2009) 104508; S. Gangopadhyay, A. E. Masunov, E. Poalelungi, and M. N. Leuenberger, J. Chem. Phys. 132 (2010) 244104.

7. C. Angeli and M. Pastore, J. Chem. Phys. 134 (2011) 184302.

8. R. Pariser and R. G. Parr, J. Chem. Phys. 21 (1953) 466; J. Chem. Phys. 21 (1953) 767; J. A. Pople, Trans. Farad. Soc. 49 (1953) 1375; Proc. Phys. Soc. 68 (1954) 81.

9. D. Zhang, Z. Qu, C. Liu, and Y. Jiang, J. Chem. Phys. 134 (2011) 024114.

10. D. Zhang and C. Liu, J. Chem. Phys. 135 (2011) 134117.

11. M. Schmidt and P. Tavan, J. Chem. Phys. 136 (2012) 124309.

12. S. Mazumdar and S. N. Dixit, Phys. Rev. Lett. 51 (1983) 292.

13. G. Konig and G. Stollhoff, Phys. Rev. Lett. 65 (1990) 1239.

14. D. Baeriswyl, D. K. Campbell, and S. Mazumdar, Conjugated Conducting Polymers, H. G. Kiess (Ed.), Springer-Verlag, Berlin, 1992.

15. W. Barford, Electronic and Optical Properties of Conjugated Polymers, Oxford Science Publications, Oxford, 1995.

16. T. G. Schmalz, Valence Bond Theory, Chapt. 17, D. L. Cooper (Ed.), Elsevier, Amsterdam, 2002.

17. S. Ramasesha and Z. G. Soos, Valence Bond Theory, Chapt. 20 , D. L. Cooper (Ed.), Elsevier, Amsterdam, 2002.

18. N. Mataga and K. Nishimoto, Z. Phys. Chem. 13 (1957) 140.

19. K. Ohno, Theor. Chim. Acta 2 (1964) 219.

20. Handbook of Chemistry and Physics, $78^{\text {th }}$ Edition, D. R. Lide (Ed.), CRC, Boca Raton, 1997.

21. For the original experimental references see M. Schreiber, M. R. Silva-Junior, S. P. A. Sauer, and W. Thiel, J. Chem. Phys. 128 (2008) 134110.

22. G. Fano, F. Ortolani, and L. Ziosi, J. Chem. Phys. 108 (1998) 9246.

23. W. Forner, C. L. Wang, F. Martino, and J. Ladik, Phys. Rev. B37 (1988) 4567.

24. R. Pariser, J. Chem. Phys. 21 (1953) 240.

25. C. E. Moore, Atomic Energy Levels vol. 1, Circular of the National Bureau of Standards 467, June 15, 1949.

26. T. G. Schmalz and L. L. Griffin, J. Chem. Phys. 131 (2009) 224301.

27. W. Haugen and M. Traetteberg, Acta Chem. Scand. 20 (1966) 1726.

28. R. D. Suenram, B. H. Pate, A. Lesarri, J. L. Neill, S. Shipman, R. A. Holmes, M. C. Leyden, and N. C. Craig, J. Phys. Chem. A 113 (2009) 1864; N. C. Craig, Y. Chen, H. A. Fuson, H. Tian, H. van Besien, A. R. Conrad, M. J. Tubergen, H. D. Rudolf, and J. Demaison, J. Phys. Chem. A 117 (2013) to appear.

29. N. C. Craig, H. Tian, and T. A. Blake, J. Phys. Chem. A 116 (2012) 3148. We note that the bond lengths given in Figure 1 of this paper for hexatriene are for the MP2 not B3LYP method. When the B3LYP values of $1.337,1.445$, and $1.346 \AA$ are inserted the theoretical end bond lengths do increase in the sequence butadiene, hexatriene, octatetraene, in contrast to the experimental result shown in Figure 8 of the paper.

30. V. M. Garcia, R. Cabollol, and J. P. Malrieu, Chem. Phys. Lett. 261 (1996) 98. 